International Journal of Bifurcation and Chaos, Vol. 13, No. 3 (2003) 685-690

(c) World Scientific Publishing Company

\title{
A RETURN MAP REGRESSION APPROACH FOR NONCOHERENT DETECTION IN CHAOTIC DIGITAL COMMUNICATIONS
}

\author{
CHI K. TSE* and FRANCIS C. M. LAU ${ }^{\dagger}$ \\ Department of Electronic and Information Engineering, \\ The Hong Kong Polytechnic University, Hong Kong, China \\ *encktse@polyu.edu.hk \\ †encmlau@polyu.edu.hk
}

Received September 2, 2001; Revised January 12, 2002

\begin{abstract}
Chaos-based communications can be applied advantageously if the property of chaotic systems is suitably exploited. In this Letter a simple noncoherent detection method for chaos-shift-keying (CSK) modulation is proposed, exploiting some distinguishable property of chaotic maps for recovering the digital message. Specifically, the proposed method exploits the difference in the return maps of the signals representing the digital symbols. The determining parameter of the return maps is estimated using a simple regression algorithm. If the parameter strongly characterizes the chaotic map, the detection can achieve very good accuracy. This strong parametric characterization can be achieved by defining the regression model with only one parameter. Using the tent maps as chaos generators, the bit-error-rate under additive white Gaussian noise is studied by computer simulations.
\end{abstract}

Keywords: Digital communication; chaotic signals; chaos-shift-keying modulation; noncoherent detection; return map; regression.

\section{Introduction}

In a chaos-shift-keying (CSK) communication system, $M$ digital symbols are represented by chaotic signals generated from $M$ dynamical systems or from one system with $M$ different parameter values [Dedieu et al., 1993; Kennedy \& Kolumbán, 2000; Kolumbán et al., 1997; Yu \& Leung, 2000]. In the binary case, i.e. $M=2$, the transmitted signal essentially switches between two chaotic signals, which are generated from two dynamical systems or from one dynamical system having a parameter switched between two values, according to the digital symbol to be represented. Detection can take either a coherent form or a noncoherent form. In coherent detection, the receiver is required to reproduce the same chaotic signals sent by the transmitter, often through a "chaos synchronization" process which is unfortunately a fragile process [Chen \& Yao, 2000]. Once reproduced, the digital symbols can be recovered by standard correlation detection [Kolumbán, 2000; Hasler \& Schimming, 2000; Suschchik et al., 2000]. In non-coherent detection of CSK, however, the receiver does not have to reproduce the chaotic signals. Rather, it makes use of some distinguishable property of the chaotic signals to determine the identity of the digital symbol being transmitted. The most commonly exploited distinguishable property has been the bit energy [Kolumbán et al., 1997; Kennedy \& Kolumbán, 2000]. However, when bit energy is chosen as the distinguishable property, detection can be accomplished easily by intruders, jeopardizing the security of the system.

It has been shown by Schimming and Hasler [2000] for a differential CSK system that suitable exploitation of the determinism of the chaotic signals 
can lead to improved performance. In this letter we consider noncoherent detection of CSK, and in particular we make use of the built-in determinism of the chaotic signals for demodulation. Specifically, the return map will be employed for detection, and our algorithm involves estimating a "strong" parameter of the chaotic map from the noisy return map received. The estimation procedure essentially follows a simple regression method.

It is of interest to note that a maximum likelihood approach for noncoherent CSK, which has been recently proposed by Kisel et al. [2001], achieves comparable performance with our proposed deterministic approach. However, our method enjoys simplicity of implementation.

\section{The CSK System}

We consider a simple CSK system, in which the transmitter sends a signal consisting of chaotic signals extracted from two chaos generators. Essentially, the signal being transmitted toggles itself between the two sequences, $\left\{a_{n}\right\}$ and $\left\{b_{n}\right\}$, depending upon the value of the digital message to be sent, where $n$ is the integer index for the sequence of values generated by the chaos generators, as shown in Fig. 1. In general, the two chaotic sequences are generated by two chaotic maps, $f: S \rightarrow S$ and $g: S \rightarrow S$ for $S \subset \Re$, s.t.,

$$
\left\{\begin{array}{l}
a_{n+1}=f\left(a_{n}\right) \\
b_{n+1}=g\left(b_{n}\right)
\end{array}\right.
$$

Our proposed system requires the chaotic maps to be written in a common form $h($.$) , with one distin-$ guishing parameter $p$, i.e.

$$
\left\{\begin{array}{l}
\tilde{a}_{n+1}=h\left(p, \tilde{a}_{n}\right) \\
\tilde{b}_{n+1}=h\left(-p, \tilde{b}_{n}\right)
\end{array}\right.
$$

where $\left(\tilde{a}_{n}, \tilde{a}_{n+1}\right)$ and $\left(a_{n}, a_{n+1}\right)$ are related by a simple transformation $T: S^{2} \rightarrow S^{\prime 2}$, and so are $\left(\tilde{b}_{n}, \tilde{b}_{n+1}\right)$ and $\left(b_{n}, b_{n+1}\right)$. That is,

$$
\begin{gathered}
\left(\tilde{a}_{n}, \tilde{a}_{n+1}\right)=T\left(a_{n}, a_{n+1}\right) \\
\left(\tilde{b}_{n}, \tilde{b}_{n+1}\right)=T\left(b_{n}, b_{n+1}\right)
\end{gathered}
$$

As will become apparent, this requirement permits a simple regression procedure to be applied to the return map for effective detection. In particular, we consider the simple case where the parameter

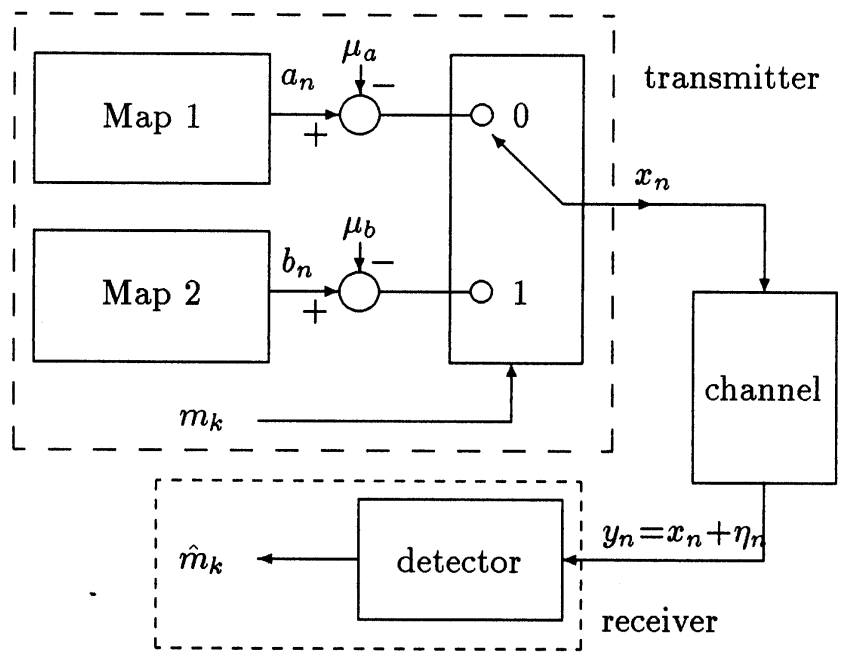

Fig. 1. Block diagram of the CSK system. $m_{k}$ is the digital message and $\hat{m}_{k}$ is the restored message. $\mu_{a}$ and $\mu_{b}$ are the means of the chaotic signals $a_{n}$ and $b_{n}$. $\eta_{n}$ denotes white Gaussian noise.

$p$ appears explicitly as a multiplier to a common function $H($.$) , i.e.$

$$
\left\{\begin{array}{l}
\tilde{a}_{n+1}=p H\left(\tilde{a}_{n}\right) \\
\tilde{b}_{n+1}=-p H\left(\tilde{b}_{n}\right)
\end{array}\right.
$$

As an example, consider the chaotic maps employing the tent maps $f(x)=1-2|x-(1 / 2)|$ and $g(x)=2|x-(1 / 2)|$ for all $x \in S=(0,1)$. The corresponding common form is given by

$$
H(x)=\frac{1}{2}-|2 x|
$$

and $p=1$. Here, the transformed domain and range are $S^{\prime}=(-1 / 2,1 / 2)$. See Fig. $2(\mathrm{a})$.

Likewise, for the logistic maps $f(x)=4 x(1-x)$ and $g(x)=4-4 x(1-x)$, with $S=(0,1)$, the corresponding common form, with $S^{\prime}=(-1 / 2,1 / 2)$, is

$$
H(x)=\left(x-\frac{\sqrt{2}}{4}\right)\left(x+\frac{\sqrt{2}}{4}\right)
$$

and $p=4$ in this case. See Fig. 2(b).

Now, consider the modulation process. Let $m_{k}$ denote the $k$ th bit, which is either 0 or 1 . Also let $N$ be the spreading factor. The modulation proceeds as follows. In each bit duration, $N$ consecutive values of either $\left\{a_{n}\right\}$ or $\left\{b_{n}\right\}$ are sent, depending upon the value of $m_{k}$. The output of the transmitter, $x_{n}$, during the $k$ th bit duration, i.e. for $n=(k-1) N+1$, $(k-1) N+2, \ldots, k N$, is given by

$$
x_{n}=\left\{\begin{array}{lll}
a_{n}-\mu_{a}, & \text { if } & m_{k}=0 \\
b_{n}-\mu_{b}, & \text { if } & m_{k}=+1
\end{array}\right.
$$




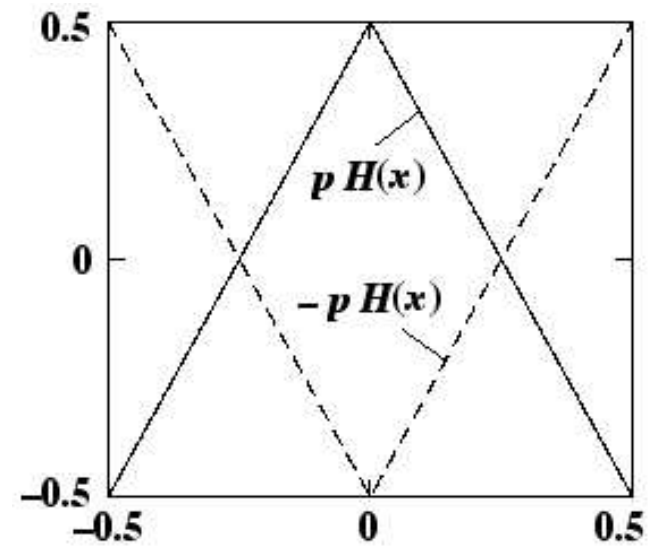

(a)

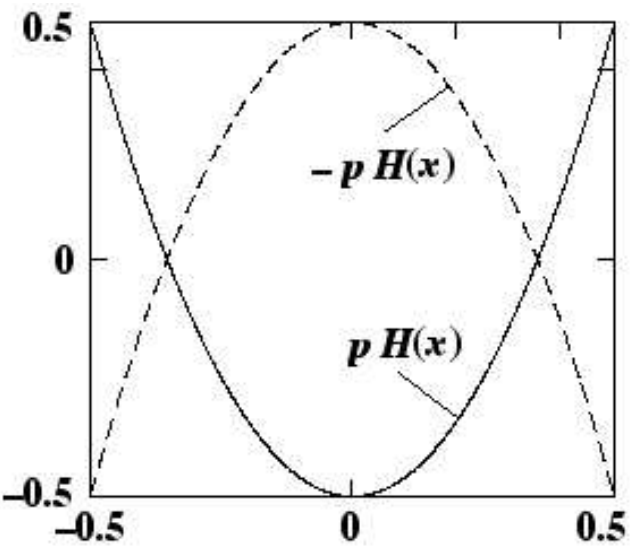

(b)

Fig. 2. Chaotic maps. (a) $H(x)=0.5-|2 x|, p=1$; (b) $H(x)=(x-\sqrt{2} / 4)(x+\sqrt{2} / 4), p=4$.

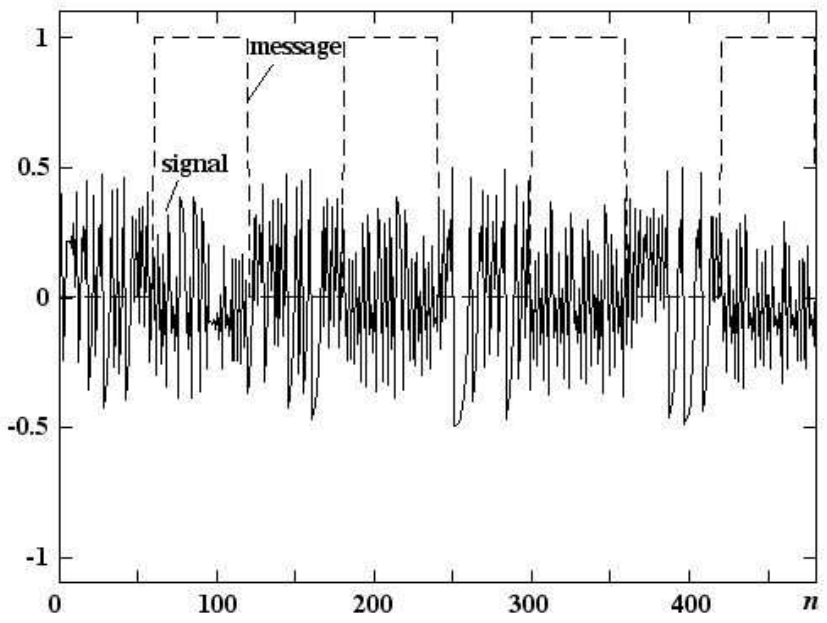

(a)

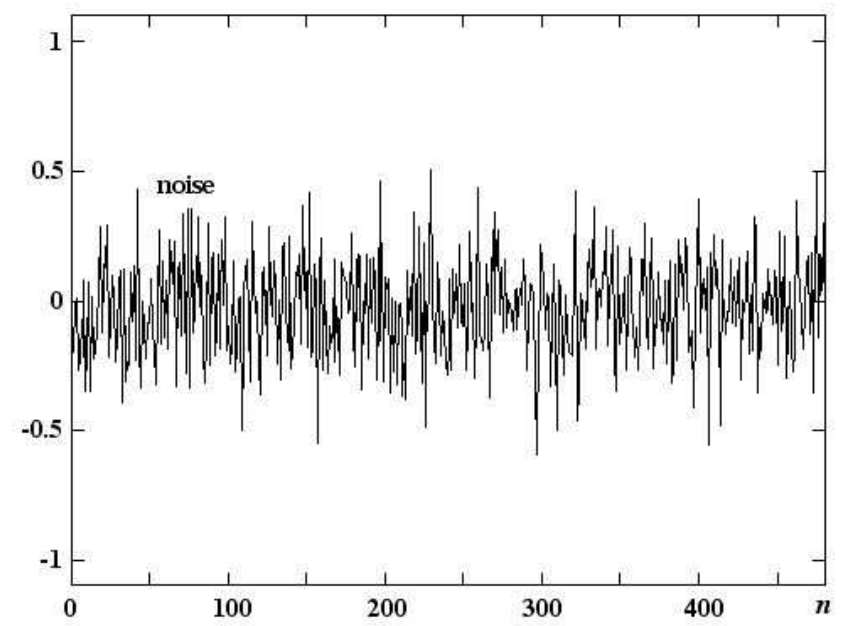

(b)

Fig. 3. Waveforms of (a) transmitted signal, and (b) channel noise at $E_{b} / N_{o}=15 \mathrm{~dB}$. Message is "01010101..." and spreading factor is 60 . The chaos generators are the tent maps $f(x)=1-2|x-(1 / 2)|$ and $g(x)=2|x-(1 / 2)|$.

where $\mu_{a}$ and $\mu_{b}$ are the average values of the two chaotic sequences $\left\{a_{n}\right\}$ and $\left\{b_{n}\right\}$, respectively. Thus, the transmitter output, $x_{n}$, has a zero average. Further, assuming that the channel is subject to additive white Gaussian noise, the signal at the input to the receiver, $y_{n}$, is given by

$$
y_{n}=x_{n}+\eta_{n}
$$

where $\eta_{n}$ is the added channel noise. At the receiving end, the general aim is to recover $m_{k}$ with a minimum probability of error.

\section{Noncoherent Detection Based on Return Map Regression}

Figure 5 shows a block diagram of the detector. Our detection approach involves first collecting the points $\left(y_{n}, y_{n+1}\right)$ for each bit. Then, the $T$ transformation mentioned in the previous section is performed on these points, such that the resulting return map resembles either the curve $y=p H(x)$ or $y=-p H(x)$ under noise-free transmission, depending upon the transmitted bit $m_{k}$ being " 0 " or "1". It should be noted that since the transmitter (as defined above) already removes the dc offset, 


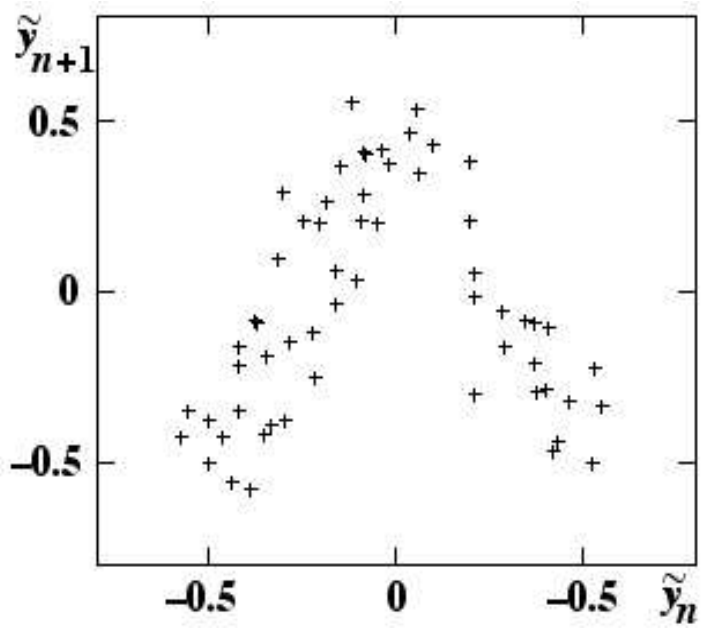

Fig. 4. Return map of received signal for symbol "0" at $E_{b} / N_{0}=25 \mathrm{~dB}$. Spreading factor is 60 .

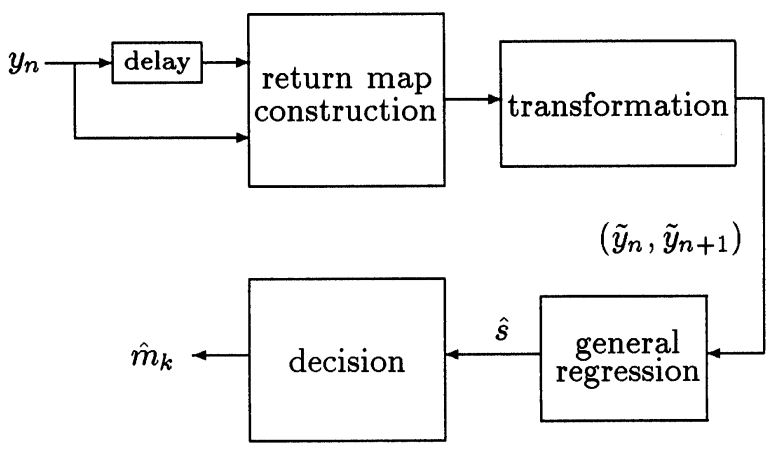

Fig. 5. Block diagram of the proposed return-map-based detector. $\hat{s}$ is the estimated slope of the transformed return map.

the transformation may need to be adjusted to take into account the dc offset. For the two examples given above, no transformation is needed because it is automatically done with the removal of the dc offset.

Under noisy condition, the points on the collected and transformed return map appear scattered, but to a certain extent (depending upon the noise level) remain close to the curves $y= \pm p H(x)$. Typical waveforms and return maps received are shown in Figs. 3 and 4 . Our detection is formulated on the basis of a regression algorithm which aims to find the best fit of the curve

$$
y=q_{k} H(x)
$$

to the points of the return map for the $k$ th bit. The regression here is to estimate the parameter $q_{k}$ such that the set of points is closest to the above curve

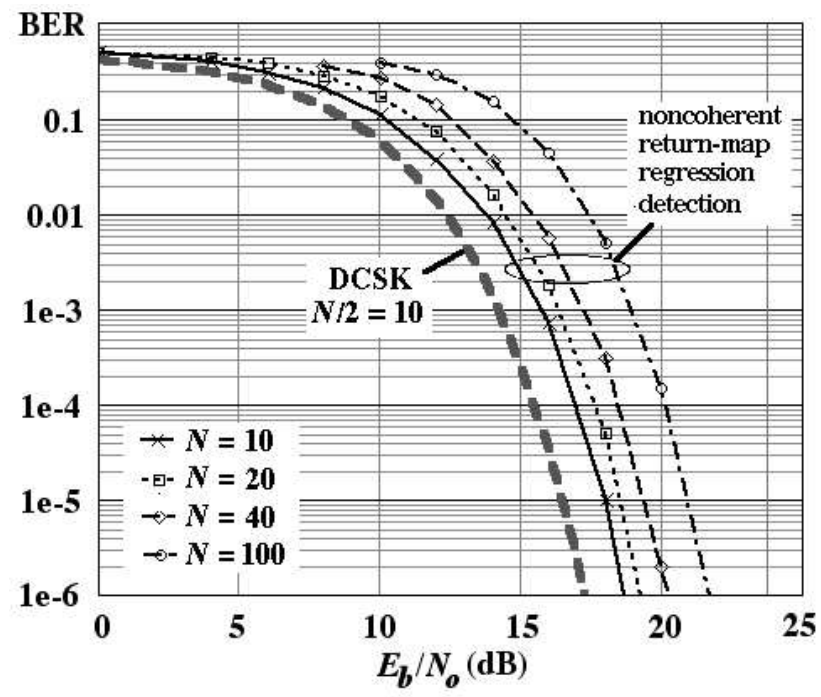

Fig. 6. BER (log scale) versus $E_{b} / N_{o}$ using the proposed return-map-based detection. Spreading factor $N=10,20$, 40 and 100. Thick dash line corresponds to DCSK with $N / 2=10$ which doubles the bandwidth requirement of our $N=10$ case.

in a least-square sense (see Appendix). Once $q_{k}$ is found, the decision rule can be as simple as

$$
m_{k}= \begin{cases}0 & \text { if } q_{k}>0 \\ 1 & \text { otherwise }\end{cases}
$$

Remarks. The essence of the transformation $T$ is to allow the two chaotic maps to be written with only one parameter which "strongly" characterizes the map. If the map is written in terms of two or more parameters, then these parameters will jointly characterize the map. Thus, the estimated value of any one parameter may not provide sufficient characterization of the particular chaotic map in order to allow accurate decision to be made as to which map has been sent. Hence, with only one parameter characterizing the map, the detection can be more accurately done.

\section{Simulation Results and Comparison}

The performance of the proposed detection method is evaluated by computer simulations. We use the simple tent maps for chaos generation, and we present here the bit error rate of the system for a range of spreading factors, i.e. $N=10,20,40$ and 100. Figure 6 shows the plots of the bit error rate 


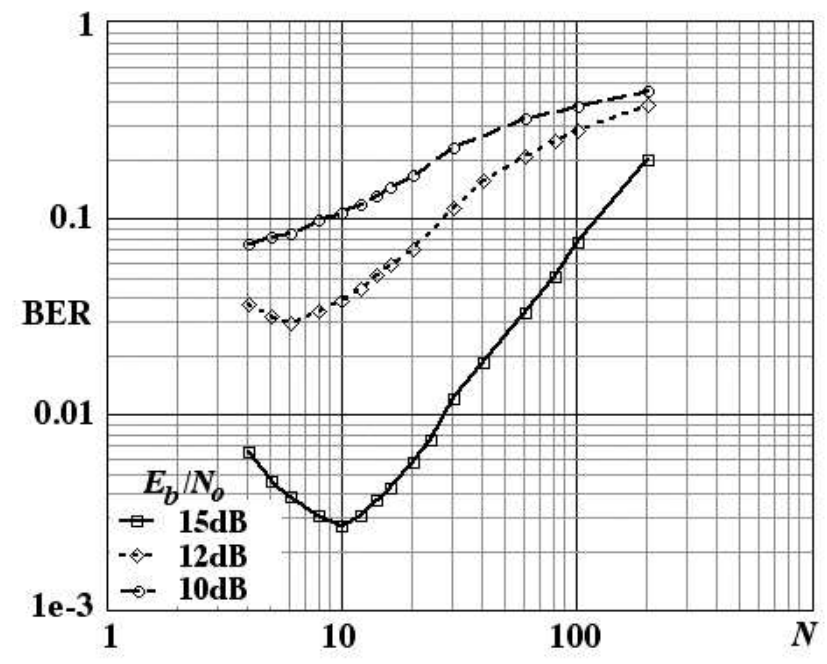

Fig. 7. Dependence of BER upon spreading factor $N$. The tent maps with $H(x)=0.5-|2 x|$ are used as chaos generators.

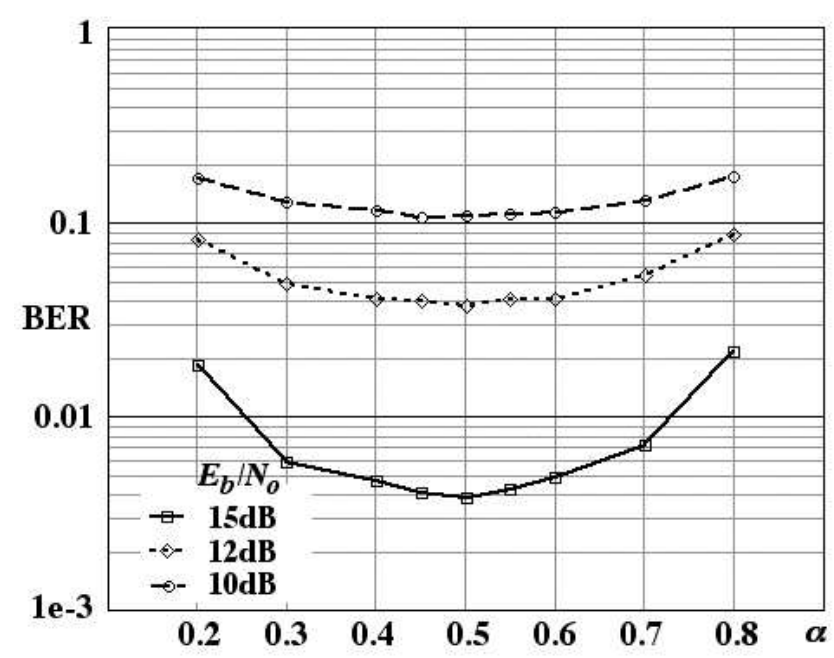

Fig. 8. Dependence of BER upon the choice of chaotic maps. The skew tent maps, with different $\alpha$, are used as chaos generators.

(BER) versus the usual $E_{b} / N_{o}$, which is defined as

$$
\frac{E_{b}}{N_{o}}=\frac{\frac{1}{N_{b}} \sum_{n=1}^{N_{b} N} x_{n}^{2}}{\frac{2}{N_{b} N} \sum_{n=1}^{N_{b} N} \eta_{n}^{2}}=\frac{N}{2}\left(\frac{\sigma_{s}^{2}}{\sigma_{n}^{2}}\right)
$$

where $N_{b}$ is the number of bits simulated, $N$ is the spreading factor (number of chips per bit), $\sigma_{s}^{2}$ is the signal power, and $\sigma_{n}^{2}$ is the noise power. The optimum performance of the noncoherent DCSK system with $N=20$ is also shown for comparison [Abel et al., 1998]. This should be compared to our $N=10$ case since half of the chips in DCSK are used as reference [Kisel et al., 2001]. Moreover, the DCSK doubles the bandwidth requirement.

Next, we study the effect of varying the spreading factor $N$. From the simulation results, we may conclude that for constant $E_{b} / N_{o}$, the BER reaches a minimum for a certain spreading factor, as shown in Fig. 7.

Moreover, the choice of chaotic maps is also important. It is interesting to compare the performance for the cases where the skew tent maps are used as chaos generators, i.e.

$$
\begin{aligned}
& f(x)= \begin{cases}\frac{x}{\alpha}, & \text { if } 0<x<\alpha \\
-\frac{1}{1-\alpha}(x-1), & \text { if } \alpha \leq x<1\end{cases} \\
& g(x)= \begin{cases}-\frac{1}{\alpha}(x-\alpha), & \text { if } 0<x<\alpha \\
\frac{1}{1-\alpha}(x-\alpha), & \text { if } \alpha \leq x<1\end{cases}
\end{aligned}
$$

where $\alpha$ is a parameter that defines the particular skew tent map. As shown in Fig. 8, the BER can be optimized by setting $\alpha$ at 0.5 for the case of skew tent map.

It should be noted that the proposed detection represents only one particular possibility of exploiting the return map features, and it may not represent the most effective algorithm for detection. Nonetheless, our purpose is to demonstrate detection possibilities by suitably exploiting the chaotic determinism.

\section{Conclusion}

In this letter we introduce an approach for demodulating CSK signals, exploiting the built-in determinism of chaotic signals. It is of the authors' view that chaos-based communications can only be applied to the best advantage if the properties of chaos can be fruitfully exploited. We demonstrate in this letter a method using the "appearance" of the return maps as a distinguishing property for recovering the digital message carried by a CSK signal. The proposed method performs comparably to the recently reported maximum likelihood detection, but the algorithm, amounting to a simple regression procedure, is much simpler. We conclude this letter by reiterating that methods based 
on detecting deterministic properties are still not exhausted and further improvement is possible for noncoherent detection based on this category of methods.

\section{Acknowledgment}

This work was supported by a research grant funded by the Research Committee of Hong Kong Polytechnic University.

\section{References}

Abel, A., Gotz, M. \& Schwarz, W. [1998] "Statistical analysis of chaotic communication schemes," Proc. IEEE Int. Symp. Circuit System, Monterey, USA, pp. IV-465-IV-468.

Chen, C. C. \& Yao, K. [2000] "Stochastic-calculusbased numerical evaluation and performance analysis of chaotic communication systems," IEEE Trans. Circuit Syst.-I 47(12), 1663-1672.

Dedieu, H., Kennedy, M. P. \& Hasler, M. [1993] "Chaos shift keying: Modulation and demodulation of a chaotic carrier using self-synchronizing Chua's circuit," IEEE Trans. Circuit Syst.-II 40(11), 634-642.

Freund, R. J. \& Wilson, W. J. [1998] Regression Analysis: Statistical Modeling of a Response Variable (Academic Press, San Diego), pp. 434-435.

Hasler, M. \& Schimming, T. [2000] "Chaos communication over noisy channels," Int. J. Bifurcation and Chaos 10(4), 719-735.

Kennedy, M. P. \& Kolumbán, G. [2000] "Digital communication using chaos," in Controlling Chaos and Bifurcation in Engineering Systems ed. Chen, G. (CRC Press, Boca Raton, FL), pp. 477-500.

Kisel, A., Dedieu, H. \& Schimming, T. [2001] "Maximum likelihood approaches for noncoherent communications with chaotic carriers," IEEE Trans. Circuit Syst. I 48(5), 533-542.

Kolumbán, G., Kennedy, M. P. \& Chua, L. O. [1997] "The role of synchronisation in digital communication using chaos — Part I: Fundamentals of digital communications," IEEE Trans. Circuit Syst.-I 44(10), 927-936.

Kolumbán, G. [2000] "Theoretical noise performance of correlator-based chaotic communication schemes," IEEE Trans. Circuit Syst.-I 47(12), 1692-1702.
Schimming, T. \& Hasler, M. [2000] "Optimal detection of differential chaos shift keying," IEEE Trans. Circuit Syst.-I 47(12), 1712-1719.

Sushchik, M., Tsimring, L. S. \& Volkovskii, A. R. [2000] "Performance analysis of correlation-based communication schemes utilizing chaos," IEEE Trans. Circuit Syst.-I 47(12), 1684-1691.

Yu, H. \& Leung, H. [2001] "A comparative study of different chaos based spread spectrum communication systems," IEEE Int. Symp. Circuit System (Sydney, Australia), pp. III-213-III-216.

\section{Appendix Least Squares Estimate of Parameter}

The basic problem is to fit a curve of the form $y=q H(x)$ to a set of data points $\left(x_{1}, y_{1}\right)$, $\left(x_{2}, y_{2}\right), \ldots,\left(x_{n}, y_{n}\right)$. The fitting objective is to minimize the residual sum of squares, defined as

$$
\mathrm{SS}=\sum_{i=1}^{n}\left[y_{i}-q H\left(x_{i}\right)\right]^{2},
$$

where $q$ is the parameter to be estimated. From elementary calculus, we set the partial derivative of the residual sum of squares with respect to $q$ to zero [Freund \& Wilson, 1998], i.e.

$$
\frac{\partial \mathrm{SS}}{\partial q}=0
$$

Expanding this, we get

$$
\sum_{i=1}^{n} 2 H\left(x_{i}\right)\left[y_{i}-\left(q H\left(x_{i}\right)\right)\right]=0 .
$$

The estimate of $q$ is thus given by the following formula:

$$
\hat{q}=\frac{\sum_{i=1}^{n} y_{i} H\left(x_{i}\right)}{\sum_{i=1}^{n} H\left(x_{i}\right)^{2}} .
$$

